http://jmscr.igmpublication.org/home/

ISSN (e)-2347-176x ISSN (p) 2455-0450

crossref DOI: https://dx.doi.org/10.18535/jmscr/v8i11.44

Journal Of Medical Science And Clinical Research

\title{
Paediatric Cataract-Summary \& Conclusion of My Study
}

\author{
Authors \\ Dr N. Suhasini M.S. FIGS ${ }^{1}$, Dr V. Suryanarayana M.S. D.O². \\ ${ }^{1}$ Asst.Prof-KIMS \& RF, Amalapuram, ${ }^{2}$ Retd. Prof, REH, Visakhapatnam
}

\begin{abstract}
Aim: To describe and analyze the current techniques utilized in the management of paediatric cataracts and the visual out come.

Materials \& Methods A retrospective study of 68 subjects who had undergone cataract surgery during 2007-08 in Govt. REH. PMMA Lens (12 mm) was implanted in the bag for children more than 2 years. Intraocular lenses were not implanted for children aged less than 2 years. Optical correction was given.

Results/Discussions: Gender ratio is M: F 7: 1; Maximum 43.75\% is in 6-9 age group ; The mean IOL power used in these subjects is $21.72 \mathrm{D}$; Despite the continued threat of amblyopia, the visual prognosis for a child with a congenital, development or traumatic cataract has improved dramatically.

Conclusions: Role of rubella and trauma in childhood cataract should be investigated and addressed. Cataract surgery with unilateral and bilateral IOL implantation can provide a beneficial effect on final visual outcome in children who are operated-on, before abnormal foveolar function develops.

Keywords: PMMA-Poly Methyl Methacrylate; REH: Regional Eye Hospital.
\end{abstract}

\section{Summary}

This study is performed to find the ways to lessen the complications of paediatric cataract surgery. However there are some complications to paediatric surgery, which threatens the vision development. The most common complication is after cataract.

The most feared complication is Glaucoma and Uveitis. The younger the infant at surgery, the more is the secondary glaucoma, Uveitis and there is development of after cataract. The children must have surgery early in life, to avoid irreversible amblyopia.

Early diagnosis of congenital cataracts is essential in ensuring treatment, together with parental advice and support. There is a good scope for investigating them using many approaches. Many questions can be easily addressed through trials and clinical studies.

The first attempt to review paediatric cataracts in central India was done by Khandekar Rajiv et al, in 2003. There is limited information available about the long-term outcomes in children treated with modern surgical techniques especially about those treated for paediatric cataract in Visakhapatnam district of Andhra Pradesh. The aim is to improve the visual prognosis in these patients.

This study comprises a consecutive series of 48 paediatric patients with congenital or developmental cataracts who received surgery between Jan, 2007 and June, 2008 at Govt. Regional Eye Hospital in Visakhapatnam. The study design and protocol are submitted to NTR University of Health Sciences for approval. 
Patients 'demographics, cataract type, presenting symptoms, surgical intervention, postoperative visual acuity and follow-up refractive changes are recorded. Evaluation included visual assessment, anterior and posterior segment examinations.

The surgical procedures included cataract extraction, intraocular lens implantation, (Posterior capsulorrhexis and anterior vitrectomy in some cases) in most of the cases. The visual status of the eyes with cataract before and 6 weeks after surgery has been evaluated. The results thus obtained are analyzed and discussed in comparison with the existing studies in the literature.

Males are more predominantly affected than females and the ratio is $\mathrm{M}: \mathrm{F}$ 7:1, Maximum affected $(43.75 \%)$ is in 6-9 age group. Mean age is $7.94 \pm 0.75$ years, Maximum people belonged to Lower social economic group $85.42 \%$, followed by middle class people i.e. $14.58 \%$.

Maximum are congenital or developmental $60.42 \%$ followed by traumatic in $37.5 \%$. In this series, Zonular cataracts are the maximum amounting to $65 \%$ followed by total cortical cataract and anterior subscapular being $10.33 \%$ each.

Intra operative complications are seen only in $16.5 \%$ of the cases, commoner being difficult rhexis in $6.08 \%$, intra operative miosis and wound leak $4.17 \%$ each and least is PC Tear in $2.08 \%$. PCO is the major and most common post operative complication amounting to $60 \%$, next being Amblyopia 30\%.

Pupillary captures of IOL and Uveitis have occurred in $4.17 \%$ and $6.08 \%$ of cases respectively. Many $29.17 \%$ of cases could get visual acuity $<3 / 60$ and another $27.25 \%$ could get visual acuity between $3 / 60$ to $6 / 60$. In $16.67 \%$ of the subjects the vision could get between $6 / 60$ to $6 / 18$. In another major chunk of subjects i.e. $20.83 \%$ vision could not be assessed.

The results of the current study are in accordance with the findings of other workers observations existing in the literature. Any minor deviation may be due to small sample size, sampling error as well as due to geographical variation.

Table No. 1 Gender distribution in the present sample

\begin{tabular}{|l|c|c|}
\hline Sex & $\mathrm{n}$ & Percentage (\%) \\
\hline Male & 42 & 87 \\
\hline Female & 6 & 13 \\
\hline Total & 48 & 100 \\
\hline
\end{tabular}

Table No. 1 and figure No.1 depicts the gender distribution of the subjects in the present sample. It is obvious that males are more predominately affected than females and the ratio is M: F 7: 1

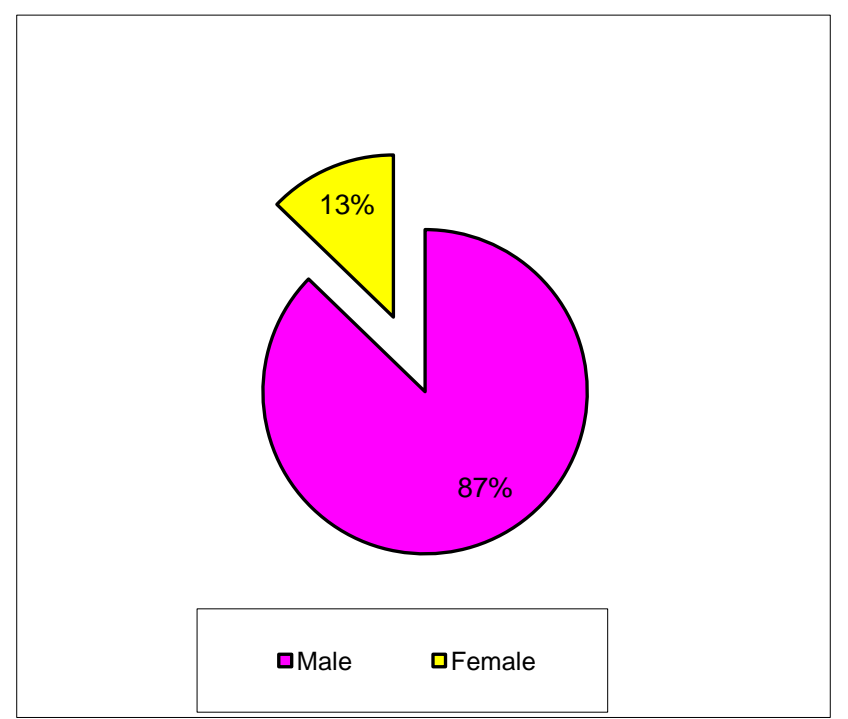

Figure No.1 Gender distribution in the present sample

Table No.2 Frequency distribution of Age

\begin{tabular}{|l|c|c|}
\hline Age groups & Number of children $(\mathrm{n})$ & Percentage $(\%)$ \\
\hline$<2$ years & 2 & 4.17 \\
\hline 2 to $5 \mathrm{yr}$ & 9 & 18.75 \\
\hline $6-9 \mathrm{yr}$ & 21 & 43.75 \\
\hline
\end{tabular}

The frequency distribution of different age groups in the present sample of paediatric cataract are shown in table No. 2 and figure No. 2. Accordingly maximum $43.75 \%$ is in 6-9 age group(school going children), followed by $10-16$ year (adolescent) age group 33.33\%, $18.75 \%$ in 25 years age group and least being below 2 years age group $4.17 \%$ 


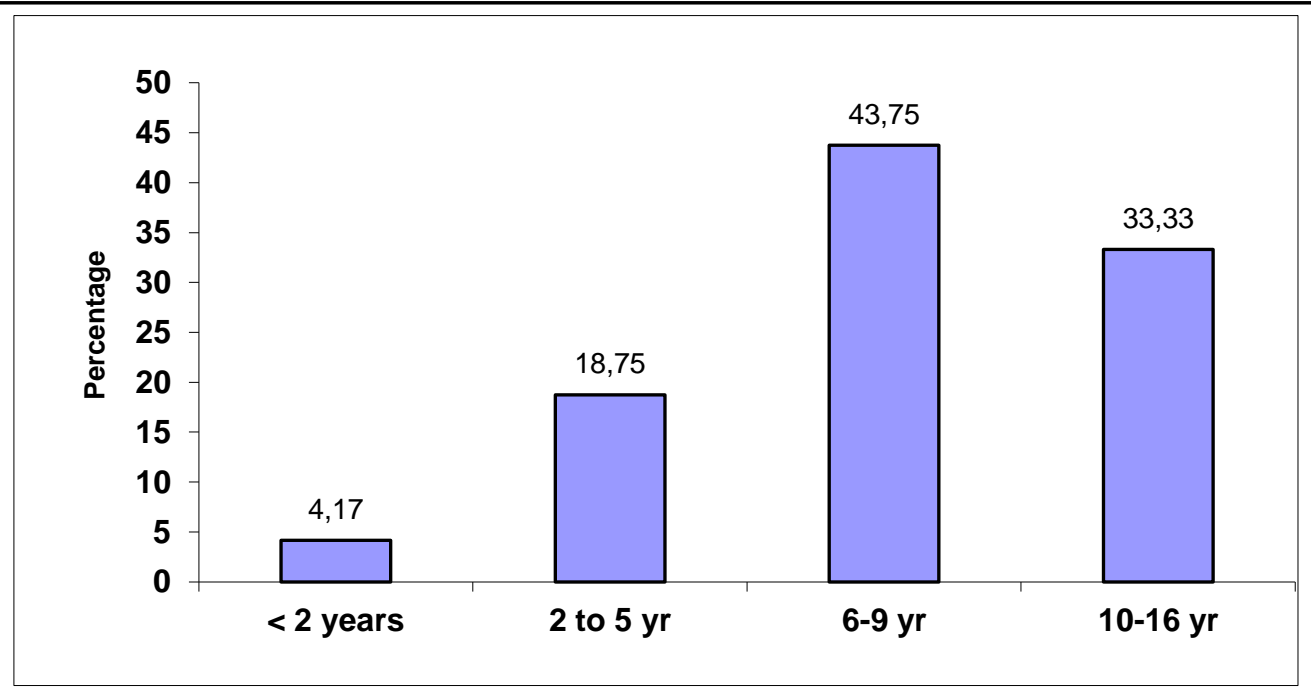

Figure No.2 Frequency Distribution of age

Table No 4 IOL Power - Descriptive Statistics

\begin{tabular}{|c|c|}
\hline IOL power & Diopters \\
\hline Mean & 21.72 \\
\hline Median & 21.00 \\
\hline Mode & 20.00 \\
\hline
\end{tabular}

The mean IOL power used in these subjects is $21.72 \mathrm{D}$, median being $21 \mathrm{D}$ and mode is $20 \mathrm{D}$ as shown in table No. 4

\section{Conclusions}

1. Cataract is more common in male children (87\%) compared to females.

2. Cataract is more common in lower socioeconomic status in the age group of 6-9 Yrs. $(43.75 \%)$

3. Bilateral congenital and developmental cataracts of Zonular type is found to be more common in our study.

4. Unilateral cataracts, Bilateral cataracts where the management has been delayed or associated nystagmus and strabismus has set in, have a poor visual outcome.

5. Primary posterior chamber Intraocular lenses are recommended for children 2 Yrs. Of age and older.

6. Most common complication is posterior capsular opacification, managed with YAG laser capsulotomy in most of the cases.
7. Early surgery combined with appropriate refractive correction and aggressive amblyopia therapy provides good visual outcome.

\section{Bibliography}

1. Outcome of cataract surgery with primary intraocular lens implantation in children Jagat Ram, Nishant Gupta, Jaspreet Singh Sukhija, Manish Chaudhary, Neelam Verma, Sunil Kumar, Saurabh Severia 2011-Bjo.Bmj.com

2. Bilateral paediatric cataract surgery in the same session $\mathrm{Y}$ Totan, $\mathrm{H}$ Bayramlar \& $\mathrm{H}$ Y1lmaz -Eye,2009-nature.com Paediatric cataract Reviewed by Jagat Ram

3. P. Nucci, editor. Karger: Basel, Switzerland- Indian journal of medical research-2018-ncbi.nlm.nih.gov

4. Outcome of paediatric cataract surgery with primary posterior capsulotomy and anterior vitrectomy using intra- operative preservative- free triamcinolone acetonide, Rohit Gupta ,Jagat Ram , Jaspreet Sukhija -Acta ophthalmologica2014

5. The Paediatric Cataract Register (PECARE): an overview of operated childhood cataract in Sweden and Denmark Gunilla Magnusson, Birgitte Haargaard, Saima Basit, Alf Nyström, 
Annika Rosensvärd, Kristina Tornqvist, Acta ophthalmologica-2018

6. Long- term results of bilateral congenital cataract treated with early cataract surgery, aphakic glasses and secondary IOL implantation, Dong- Hyun Kim, Jeong Hun Kim, Seong- Joon Kim -Acta Ophthalmologica-2012

7. Accuracy of routine data on paediatric cataract in the UK compared to active surveillance: lessons from the IOLu2 study Ameenat Lola Solebo1, Isabelle RussellEggitt2, JugnooSangheeta Rahi1,2, British journal of Ophthalmology-2013

8. Outcome of paediatric cataract surgery in Northwest Ethiopia: a retrospective case series Mulusew Asferaw1, SisayYoseph Mekonen1, Geoffrey Woodruff2, Clare E Gilbert3, Samson Tesfaye4 -British journal of Ophthalmology-2019. 